\title{
Meaning in Human Ecology
}

\author{
Philip J. Stewart \\ Commonwealth Forestry Institute \\ University of Oxford, Oxford, United Kingdom
}

No one would describe the elephant without its trunk nor the kangaroo without its jump, yet much of what passes for scientific description of our species ignores its most striking feature-an extraordinary capacity for finding and making patterns. Of special importance is the peculiar pattern of relationships between patterns that is called meaning.

The word "meaning" is commonly thought of as referring in its strict sense only to language; expressions like "the meaning of a certain smile" or, still more, "the meaning of your life" are regarded as metaphor. However, linguistic meaning is only a special case; more generally, meaning can be defined as the association in a nervous system of certain patterns (signs) with others that they call up (referents). However, this definition seems to cover the whole of mental life, for the whole content of our minds is made up of the association of patterns. More precision is needed.

At its elementary level, meaning is simply part of the process by which any organism with a nervous system knows its environment. Certain patterns are found to recur regularly in association with others, of which they become the signs. In this way, for example, the experienced lion knows where its prey are, not by seeing them directly but by finding the signs of their passage. In so far as the patterns are naturally connected, this may be called inherent meaning. In a sense we never perceive anything but signs, inferring from patterns of light and sound, scent and touch, the objects we thus suppose to underlie them. Even at this level, there is a syntax of signs, two or more coming together to mean what none of them would mean separately. Thus, for example, the lion learns the combination of patterns that make up a waterhole and mean game.

Inherent meanings are all around waiting to be learned, and the mature adults of a given species end up by learning all or most of those that enable them to survive. In many species much of the repertoire is genetically transmitted. The higher animals, however, learn many signs in the course of their life, and this enables them also to pick up what may be called accidental meanings, where there is no natural or

1 This manuscript has been transcribed from the article originally published in R. J. Borden (Ed.) (1986). Human ecology: A gathering of perspectives. Bar Harbor, ME: Society for Human Ecology. While all care has been taken, minor typographical differences to the original may occur. 
necessary connection between signs and referent. There is of course no hard and fast frontier between inherent and accidental; the extent to which patterns are associated varies continuously from totally to not at all.

For animals, accidental meaning is usually peculiar to the individual. For example, a dog that has been kicked by a man with a hat may afterwards cringe from anyone wearing a similar hat, but this is not passed on to other dogs. Human beings are peculiar in their ability to transmit belief in accidental meaning to a whole cultural group, making it into intentional meaning. Patterns are arbitrarily chosen for their convenience and deliberately used as signs or symbols. It should not be thought that these are all artificial. Natural objects and events can be used as symbols; indeed, bodily functions carry a heavy burden of symbolism. Eating, for example, is hardly ever undertaken as something purely functional, for each food and drink is invested with meaning. Sex can perhaps be the most potent symbol of all, which leads to frequent conflict between the meaningful and biologically desirable.

Each culture except the simplest surrounds its members with a wealth of patterns out of which to read meaning. Clothes and hairstyles, tools and utensils, toys and games, art and music, houses and gardens, roads and vehicles all contribute to making visible the social pattern into which each person fits. The demand for food and materials thus created shapes the landscape in which people live. This cultural world is already there, waiting for each new child who is born into it, and so it seems like something given and not like the sum of countless attempts to express meaning.

The most complex, flexible, and versatile system of symbols is language, which has been found fully developed in every human society ever described, however simple its material culture. The achievement of Washoe and Sarah, the signaling chimpanzees, showed an unexpected ability to convey intentional meaning, and the communication of dolphins and whales probably has surprises in store for us; but human linguistic capacity undoubtedly far exceeds that of any other species. Starting with nothing but observation of the sound patterns associated with different contexts, each child in a few short years learns thousands of words and the rules of syntax implicit in hundreds of sentence patterns.

With mastery of language comes entry into the social environment of the people who use it. Slight differences in speech act as signs indicating the place of each individual in the pattern of society. Marked differences act as barriers to communication, dividing the human species into thousands of linguistic pseudospecies. The invention of writing vastly increased the potential of language, making it possible to store huge quantities of utterances. For hundreds of years - thousands in some countries-bookish education has been the way to the predominant places in society, giving prominence to people whose concern with words is often so great that they have lost much of their ability to read other kinds of signs. 
Skill in the use of words brings abstraction-the ability to describe patterns of patterns and patterns of patterns of patterns - sinking deeper and deeper beneath surface phenomena. To the ape in its forest almost every sign is part of a natural process - the reddening of a fruit, the darkening of the sky, the threatening stance of an old male - and each points to something tangible. Human beings alone can talk of quarks and black holes, of nothing and the Universe, of God and the Devil. They also have an extraordinary ability to convey half-truths, errors, lies, fantasies and nonsense. Words and symbols thus take on an apparent autonomy, seeming independent in the real world. This has given rise to a persistent dualism-the belief that pattern can exist without the thing patterned, soul without body, mind without matter, God without Universe.

It seems to be a universal characteristic of human beings to use the capacity for abstraction to reach a concept of the total pattern of all things. Traditional cultures point to this ultimate pattern with symbols such as the dance of Shiva or the meditating Buddha, and with phrases such as "God is Love" or "There is no God but Allah." Some individuals have such a strong sense of ultimate pattern that they find it reflected everywhere, being able "to see the world in a grain of sand and a heaven in a wild flower."

The most remarkable thing of all is that people of every culture have tried to make the pattern of their individual and collective lives correspond in some way with the ultimate pattern. They have imitated the life of those who are supposed to have embodied it. They have laid out their buildings and cities as little models of the cosmos and adorned them with countless symbols. They have punctuated their existence with rites and festivals that reflect universal cycles. But this correspondence of small and large pattern is precisely the relationship that is meaning. In other words, human life itself becomes a symbol—a fitting destiny for a meaning-centered species.

Attachment to ultimate pattern can be even stronger than the desire to live. Throughout history there have been examples of martyrdom, in which healthy people in the prime of life die willingly—even joyfully_making their death the final symbolic act. Conversely, those who fail to fit their life to an ultimate pattern live listlessly_even miserably—seeing their existence as meaningless, "just one damned thing after another," "a tale told by an idiot, full of sound and fury, signifying nothing." A meaningful death can thus be preferred to a meaningless life-strange behavior indeed for mere biological matter!

Shared belief in ultimate pattern has provided the framework into which all lesser symbols and beliefs are integrated. It has also enabled the members of certain societies to risk their lives in spreading their cultures at the expense of others. In very ancient times it brought Hinduism to all of India. Later on it took Christian missionaries all over western Eurasia and Buddhists all over the East. Still later it 
sent Muslim armies, settlers, and missionaries throughout Central Asia and North Africa, Protestants to North America, and Catholics to South and Central America. Most recently of all it has carried Christianity and Islam to most of Africa. The cultural geography of the world has thus been radically simplified, thousands of older versions of ultimate pattern being destroyed by—or integrated into-four major ones.

The rich world of meaning has had immense importance not only in regulating the internal affairs of the human species but also in determining its effects on the rest of the planet. Take the landscapes of the Muslim world, for example. The predominance of grazing land can be directly attributed to the sacrificial role of the sheep and other herbivores, which is traced back to Abraham's miracle of the ram in the thicket. The widespread disappearance of forests owes something also to the ban on the meat of the pig, the one domestic animal capable of thriving in woodland without destroying it. Similarly, the infrequency of vineyards, which elsewhere bring prosperity and protection to poor soils and steep slopes, results from the prohibition of alcohol. The prominence of the olive and the fig tree can be traced partly to the mention of both in the Koran. None of this can be explained in purely material terms.

In recent centuries the traditional symbols of ultimate pattern have come under sustained attack. At too many points they have, at least if taken literally, been contradicted by the findings of experimental science. At the same time, modern travel and communications have brought people face toface with traditional accounts that, on the surface, cannot be reconciled, which has led many to conclude that all versions are false. The symbolic value of things has come to be seen as something essentially private and subjective, leaving the public domain to be governed by the criterion of utility. To eyes that have seen the rich symbolism of older cultures, the utilitarian modern world can look very drab.

Deprived of an integrating framework of ultimate pattern, the various branches of knowledge and action have drifted apart. People are led to believe that life consists of an assortment of self-contained departments: politics, the economy, defense, religion, science, medicine, law, nutrition, sex, art and so on. Countless specialists work, often with large sums of public money, to change the world according to their own narrow idea of what is best, largely in ignorance of the bad effects they may have on ordinary people or on the rest of the biosphere.

The present century has seen rapid progress toward an integrated scientific account of the material world, with bridges built between the physics of the very small and the very large, between physics and chemistry, chemistry and biology. As yet, this account has not had a place for the mental world of pattern and meaning. Now that is changing. Cybernetics, information theory, and catastrophe theory have thrown light on the nature of self-patterning systems. Computing has made possible 
the simulation of highly complex phenomena. The study of cellular automata has brought a new humility in the face of that which cannot be simplified. And the discovery of genetic code has brought concepts analogous to those of linguistics into the heart of biology.

Scientists have not yet shown much interest in meaning, although the correspondence between sign and referent is often visible in behavior, in a way that should satisfy the strictest Skinnerian. They have so far preferred information, which is quantifiable as the amount of improbability present in a pattern with a given number of degrees of freedom. Great interest was aroused when it was shown by Shannon and Weaver that the concept of information is closely related to that of negentropy. Indeed, Shannon called his uncertainty function "entropy." This identity results from the fact that both are applications of the same mathematics-those of probability. Total randomness, if that is possible, would be total absence of pattern and therefore of information.

Unfortunately Shannon's information is not the same thing as the information of everyday life. It is, for example, possible to calculate the number of kilobytes in an Etruscan text; but until we know the language, it will convey no information. In other words, information in the sense of "bytage" is an intrinsic property of a pattern; information in the ordinary sense is a one-for-one correspondence between elements in two different patterns. Usually, in fact, exact correspondence is not possible, and there is approximation. Where the source pattern is capable of corresponding to more than one other pattern there is ambiguity.

The word "meaning" is sometimes used to refer to information in the ordinary sense, as in the example of "RSVP" meaning "please reply." More usually, however, there is a one-to-many or few-to-many correspondence between the elements in the sign and the elements in the referent. Put differently, the emitter and the receiver of a sign must already possess information about the referent. For example, a vast pattern of political events could be translated into a single bit of information-say the turning on of a switch-which in turn would mean to its military recipients all the events rehearsed for the start of a war. In terms of bytage, meaning can thus be far more concentrated than information.

From the switch that means war up to the dance of Shiva that means the way of the Universe and down to the waterhole that means game, there is a unifying concept of meaning that can now be scientifically approached, thanks to advances in the understanding of pattern. So far only anthropologists seem to have given meaning its rightful place. For too long academic psychologists have been exclusively concerned with observable behavior, sociologists with structure and function, economists with utility and efficiency. Human beings have thus been studied as if they were little more than ants or rats and devoid of their most precious faculty. 
Academic human ecology has so far failed to give pattern and meaning a central place. For historical reasons ecology, the study of the relations of species with the external environment, grew up separately from ethology, which studies the relations of members of each species with the internal environment of their own society, in which most signaling behavior takes place. A similar distinction was observed when the ideas of ecology came to be applied to human beings. In recent years, however, it has become increasingly plain to biologists that the two sets of relations cannot properly be separated. For example, the reproductive behavior of a species, which constitutes much of its ethology, is closely related to its population structure and its demands on the ecosystem.

It is time to recognize that human ecology must include the human equivalent of ethology, and that our relationships with the rest of the world can only be understood in the light of our relationships with each other. The insights of the psychologist, the anthropologist, the historian, the linguist, and the critic of art and literature are as necessary as those of the physiologist, the geneticist, or the demographer. Human ecology, in other words, is a framework for synthesis rather than just another discipline parallel with the rest.

Within this framework, the mental and physical both have their place, with pattern and meaning as the unifying concepts. This implies a view of the world distinct at once from materialism, which denies the reality of the mental, from idealism, which refuses that of the physical, and from dualism, which sees the two as essentially separate. Such an approach is in keeping with modern physics, which sees matter as a pattern of atoms, atoms as a pattern of electrons and nucleons, nucleons as a pattern of quarks, quarks and electrons as a pattern of energy, energy as a pattern of disturbances in a field ... in short, a Universe of patterns of patterns of patterns ...

An integrated human ecology offers more than just an extension of knowledge. By providing a comprehensive model of human relationships with the intricately patterned physical world, it points the way to a scientifically acceptable account of ultimate pattern, within which people may find the meaning of their lives. The exploits of Greenpeace suggest that an ecological account is as capable as the traditional ones of inspiring people to risk their lives.

Many of the ancient myths and symbols of ultimate pattern are consonant with an ecological view. This is not to suggest that all traditional accounts can be fused into some vague synthesis, still less that they are mere forerunners of modern secular ideas. Each is partly the reflection of its own social matrix and inappropriate to others. At their worst they can be crassly dualist or idealist. At their best they reach heights of incomparable sublimity. Always they are in danger of being understood literally instead of metaphorically; but they also go with techniques such as meditation that can be more effective than words for achieving harmony between mind and larger patterns. 
In the Indian parable of the blind people trying to describe an elephant, the one who felt at the trunk said: "This animal is like a snake." In the same way it would be only part of the truth to say of our species: "This creature is like a string of signs." A full account must also include all the other aspects of human life. Even if we human ecologists succeed in making such a synthesis, we must keep a sense of humility. Like the blind in the parable, we shall never achieve a description that is complete or final or free from error and dispute. In the words of the Tao Te Ching: "Knowable pattern is not ultimate pattern."

\section{Acknowledgments}

My thanks are due to the Commonwealth Human Ecology Council, which gave support in the preparation of this paper and which helped, altogether with the Commonwealth Forestry Institute, to make it possible for me to attend the meeting of the Society for Human Ecology. 
This text is taken from Human Ecology Review, Volume 23, Number 2, 2017, published 2017 by ANU Press, The Australian National University, Canberra, Australia.

dx.doi.org/10.22459/HER.23.02.2017.13 